\title{
Redefining the Role of Cox-2 in Human and Canine Malignancies
}

\author{
Naresh Kumar Sood ${ }^{1 *}$ and Ruhi Sood ${ }^{2}$ \\ ${ }^{1}$ Department of Teaching Veterinary Clinical Complex, Guru Angad Dev Veterinary and Animal Sciences University, India \\ ${ }^{2}$ Department of Veterinary Medicine, Szent István University, Budapest, Hungary
}

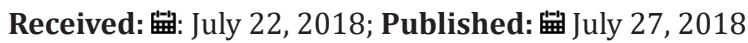

*Corresponding author: Naresh Kumar Sood, Department of Teaching Veterinary Clinical Complex, Guru Angad Dev Veterinary and Animal Sciences University, India.

Abstract

The role of COX enzymes was previously well defined in the inflammation and pain. Recently, studies have shown increased COX-2 expression in malignant tumors of the human and canine patients and its association with multiple events in cancer progression. As of now, it is likely to be a novel target of intervention by combining standard chemotherapy with NSAIDs, especially to delay the progression of cancer.

Abbreviations: Non-Steroidal Anti-Inflammatory Drug (NSAID); Cyclooxygenase (COX)

\section{Introduction}

In the 1970s and 1980s, the first series of experimentation on the constitutively expressed cyclooxygenase(COX)-1 began. However, just as interest started to diminish, the discovery of the inducible isoform, COX-2, rekindled a largely fresh effort that ultimately led to new insights about both the isoforms [1]. COX2 , traditionally associated with inflammation, has been a key target and the mainstay of non-steroidal anti-inflammatory drug (NSAID) therapy, which has been extensively used in the treatment of pain, fever, and inflammation [2]. However its role has been subsequently expanding. Currently the focus of research is about COX-2 involvement in causation and progression of cancer and its inhibition by NSAIDs [3-5].

COX-2 is a key enzyme that controls the conversion of arachidonic acid to prostaglandin( $\mathrm{PG})$. The precursor of $\mathrm{PG}$ synthesis is arachidonic acid, a 20 carbon poly-unsaturated fatty acid. The hydrolysis of phospholipids to produce free arachidonic acid is catalysed by phospholipase $A_{2}$. The next step which inserts molecular oxygen into arachidonic acid is catalysed by COX-2, the reaction of which produces an unstable product, PGG2, which is then converted by the peroxidase activity of COX to PGH2. PGH2 is the common precursor for all other prostanoids. The production of individual prostanoids is catalysed by different specific synthases, which may vary in their expression between different types of cells. Each of the products derived from $\mathrm{PGH}_{2}$ has a distinct biological function [6].

COX-2, a key enzyme in eicosanoid biosynthesis is a rate limiting factor in the synthesis of prostaglandin from arachidonic acid. Two isoforms of COX are known, COX-1, the constitutive isoform; and COX-2, the inducible isoform of the enzyme. Studies in human breast cancer patients revealed that COX-1 is expressed ubiquitously, and its role is connected to physiological functions, such as cytoprotection of the stomach and control of platelet aggregation [7]. Whereas the role of COX-2, the inducible form of the enzyme, has been connected to inflammation and carcinogenesis. COX-2 is usually undetectable in normal tissues, but undergoes rapid induction in response to cell activation by pro-inflammatory cytokines, growth factors, oncogenes, bacterial lipopolysaccharides and phorbol esters. Thus COX-2 is overexpressed in some malignancies and premalignant lesions and not COX-1 [8].

\section{COX-2 Overexpression and Carcinogenesis}

The relation between COX-2 and cancer has been studied for many years. This relationship has been postulated based on various studies, which established an association between chronic use of nonsteroidal anti-inflammatory (NSAID) drugs and decreased incidence of colorectal carcinoma shown first in human patients [9], where the role of COX-2 and prostaglandins was suspected after epidemiological studies had revealed that the regular intake of low doses of aspirin reduced the risk of colorectal cancer.

As in colorectal cancer, elevated expression of COX-2 has been reported in breast cancer in women with studies showing that approximately $40-50 \%$ of invasive breast carcinomas have high COX-2 protein levels. Carcinomas with increased COX-2 expression have been correlated with worse prognosis for women with breast cancer. In addition to colorectal cancer, many other types of human malignancies have now been shown to overexpress COX-2, such as breast, pulmonary, head and neck, pancreatic, prostatic, and gastric cancers [10]. 
Based on several experimental studies, mechanisms associated with tumoral promotion such as an increase in angiogenesis, inhibition of apoptosis, suppression and modulation of the immune response and greater invasive and metastatic capacities have been proposed to explain the consequences of COX-2 overexpression [8].

\section{COX-2 Overexpression and Its Relation to Canine Malignancies}

In veterinary medicine, COX-2 is shown to be overexpressed in a variety of tumors, including tumor of the prostate, transitional cell carcinoma, mammary gland tumors, skin and nasal tumors, among others [11]. In a study in the year 2003, it was demonstrated for the first time that COX-2 is induced in a proportion of canine mammary tumors suggesting a potential role for COX-2 in canine mammary tumorigenesis [12]. Malignant canine mammary tumors express COX-2 more strongly than benign tumors, irrespective of histologic type [13]. About $60 \%$ of canine mammary gland tumors show COX2 overexpression with most of them being malignant. Hence, the higher the expression of COX-2, the poorer the prognosis [11].

\section{COX-2 Overexpression and Its Role in Neo-Angiogenesis in Cancer}

It had been suggested over a decade ago, that COX-2 overexpression may lead to increased neo-angiogenesis, such that the inhibition of COX-2 might have a general anti-cancer effect via decreased blood vessel formation in humans [6]. This link between COX- 2 overexpression and tumor angiogenesis was established by various studies in human medicine in the following years [14], but more recently, Queiroga found a more sound correlation between tumor angiogenesis and COX-2 overexpression in canine mammary cancer [15].

COX-2 is associated with the production of vascular endothelial growth factor (VEGF), which stimulates the growth of endothelial cells, and thereby, promotes angiogenesis, which is needed for most solid tumors, as newly formed blood vessels provide nutrients for their growth and survival [16]. The use of COX-2 specific inhibitors has been suggested as a potential therapeutic tool for canine mammary gland tumor management by blocking COX-2 mediated angiogenesis pathways [17].

\section{COX-2 Overexpression and Apoptosis Inhibition in Cancer}

The inhibition of apoptosis, a process of programmed cell death, appears to be the key pathway in the survival of cancer cells. COX-2 plays an important role in tumor cell biology acting through apoptosis inibition, by promoting invasiveness and by modulating the production of angiogenic factors. It has been postulated since long that mechanisms underlying the association between COX-2 overexpression and tumorigenic potential may include resistance to programmed cell death [18].

The role of COX-2 in apoptosis has been observed in rat intestinal epithelial (RIE) cells. Studies suggest that COX-2 overexpression inhibits apoptosis by the induction of Bcl-2 expression or other members of the Bcl family (Mcl1) and that this anti-apoptotic effect can extend the survival of abnormal cells. The upregulation of Bcl-2 in tumor microvascular endothelial cells not only enhances cell survival improving the ability of these cells to remain viable and functional despite the constraints imposed by the tumor microenvironment, but also engages them in a more vigorous angiogenic response in vitro and in vivo [19].

The fact that tumors derived from cells that overexpress Bcl2 grow more aggressively in vivo has been attributed by several authors to the anti-apoptotic properties of $\mathrm{Bcl}-2$ resulting in neoplastic transformations. Also there is evidence of reduction of Bcl-2 expression and induction of apoptosis in cancer cells after the use of COX-2 inhibitors [20]. Enhanced prostaglandin synthesis may also contribute to tumorigenesis by direct stimulation of cell growth, through activation of mitogenic, mutagenic and aromatase pathways [5].

\section{Conclusion}

Significant headway has been made in our understanding of COX-2 and its role in tumorigenesis. COX-2 overexpression and prostaglandin synthesis may contribute to tumorigenesis via increased cellular proliferation, decreased apoptosis, enhanced cell invasiveness, increased mutagen production, diminished immune surveillance and effects on angiogenesis. Cyclooxygenase-2 inhibitors are relatively safe in humans and are an interesting class of drugs with potential for future cancer management, either alone or in combination with conventional therapies.

\section{References}

1. Rouzer CA, Marnett LJ (2009) Cyclooxygenases: structural and functional insights. Journal of Lipid Research 50: S29-S34.

2. Bakhle YS, Botting RM (1996) Cyclooxygenase-2 and its regulation in inflammation. Mediators of Inflammation 5(5): 305-323.

3. Hugo HJ, Saunders C, Ramsay RG, Thompson EW (2015) New Insights on COX-2 in Chronic Inflammation Driving Breast Cancer Growth and Metastasis. J Mammary Gland Biol Neoplasia 20: 109-119.

4. Bing L, Liyan $\boldsymbol{Q}$, Shigui $\boldsymbol{Y}$ (2015) Cyclooxygenase-2 promotes tumor growth and suppresses tumor immunity. Cancer Cell International 15: 106-111.

5. Chau TD, Charles LS, Clifford AH (2002) Potential Role of Selective COX-2 Inhibitors in Cancer Management. Cancer network.

6. Davies G, Martin LA, Sacks N, Dowsett M (2002) Cyclooxygenase-2 (COX2), aromatase and breast cancer: A possible role for COX-2 inhibitors in breast cancer chemoprevention. Annals of Oncology 13(5): 669-678.

7. Ristimäki A, Sivula A, Lundin J, Lundin M, Salminen T (2002) Prognostic Significance of Elevated Cyclooxygenase-2 Expression in Breast Cancer. Cancer Research 62(3): 632-635.

8. Howe LR (2007) Cyclooxygenase/prostaglandin signaling and breast cancer. Breast Cancer Research 9(4): 210-219.

9. Thun MJ, Namboodiri Mm, Heath CJ (1991) Aspirin use and reduced risk of fatal colon cancer. N Engl J Med 325: 1593-1596.

10. Doré M (2011) Cyclooxygenase-2 Expression in Animal Cancers. Vet Pathol 48(1): 254-265.

11. Queiroga FL (2008) Proceedings of the $4^{\text {th }}$ Merial European symposium on Pain management. Split, Croatia p. 79-86.

12. Doré M, Lanthier I, Sirois J (2003) Cyclooxygenase-2 Expression in Canine Mammary Tumors. Vet Pathol 40: 207-212.

13. Lavalle GE, Bertagnolli AC, Tavares WLF, Cassali GD (2009) Cox2 Expression in Canine Mammary Carcinomas: Correlation with Angiogenesis and Overall Survival. Vet Pathol 46: 1275-1280. 
14. Basu GD, Liang WS, Stephan DA, Wegener LT, Conley CR (2006) A novel role for cyclooxygenase-2 in regulating vascular channel formation by human breast cancer cells. Breast Cancer Research 8(6): R69.

15. Queiroga FL, Pires I, Parente M, Gregorio H, Lopes CS (2010) COX-2 overexpression correlates with VEGF and tumour angiogenesis in canine mammary cancer. The Veterinary Journal 88(3): 441-445.

16. Nardi ABD, Raposo TMM, Huppes RR, Daleck CR, Amorim RL (2011) Cox-2 inhibitors for cancer treatment in dogs. Pakistan Veterinary Journal 31(4): 275-279.

\section{ISSN: 2574-1241}

DOI: 10.26717/BJSTR.2018.07.001488

Naresh Kumar Sood. Biomed J Sci \& Tech Res

(C) This work is licensed under Creative

Submission Link: https://biomedres.us/submit-manuscript.php
17. Queiroga FL, Pires I, Parente M, Gregório H, Lopes CS (2011) COX-2 overexpression correlates with VEGF and tumour angiogenesis in canine mammary cancer. The Veterinary Journal 189(1): 77-82.

18. Crofford LJ (1997) COX-1 and COX-2 tissue expression: implications and predictions. The Journal of Rheumatology 49: 15-19.

19. Carmeliet P (2005) VEGF as a Key Mediator of Angiogenesis in Cancer. Oncology 69: 4-10.

20. Cao Y, Perscott SM (2002) Many Actions of Cyclooxygenase-2 in Cellular Dynamics and in Cancer. Cellular Physiology (190): 279-286.

BIOMEDICAL
RESEARCHES $\quad \begin{aligned} & \text { Assets of Publishing with us } \\ & \text { - Global archiving of articles }\end{aligned}$

